\title{
Is There Relationship between Quality Indicators and Acquired Pressure Ulcers in Austrian Hospitals and Nursing Homes
}

\author{
Eman S. M. Shahin ${ }^{1,2}$, Christa Lohrmann ${ }^{2}$ \\ ${ }^{1}$ Medical-Surgical Nursing department, Faculty of Nursing, Port Said University, Port Said, Egypt \\ ${ }^{2}$ Department of Nursing Science, Medical University of Graz, Graz, Austria \\ Email address: \\ emanshaheen@yahoo.com (E. S. M. Shahin), Christa.lohrmann@medunigraz.at (C. Lohrmann)
}

\section{To cite this article:}

Eman S. M. Shahin, Christa Lohrmann. Is There Relationship between Quality Indicators and Acquired Pressure Ulcers in Austrian Hospitals and Nursing Homes. Journal of Surgery. Special Issue: Postoperative Pain Syndrome. Vol. 3, No. 2-1, 2015, pp. 1-7.

doi: $10.11648 /$ j.js.s.2015030201.11

\begin{abstract}
Objectives: this study aims to assess the period prevalence of Austrian hospital- and nursing home-acquired pressure ulcers, and of pressure ulcer quality indicators in both settings over time. Methods: Design: A descriptive study (period prevalence) was conducted in hospitals and nursing homes between 2009 and 2012. The study sample covered hospital patients and nursing home residents who gave informed consent in the study ( $\mathrm{n}=13,438$ ). The instrument used in this study was the German version of the Dutch National Prevalence Measurement of Care Problems (Landelijke Prevalentiemeting Zorgproblemen/LPZ) which covers demographic data, questions regarding quality indicators of pressure ulcers and the Braden scale. The results revealed that the period prevalence of pressure ulcers, excluding category one, was $6.4 \%$ in hospitals and $6.3 \%$ in nursing homes, whereas the period prevalence of hospital-acquired pressure ulcers, excluding category one, was $2.4 \%$ in hospitals and $4.6 \%$ in nursing homes. Six quality indicators for pressure ulcers (e.g. pressure ulcer prevention committee, pressure ulcer agreement guidelines, management protocol/guidelines for pressure ulcer prevention products, information brochures) exist in hospitals and five in nursing homes. Conclusion: The results indicated that $44 \%$ of pressure ulcer period prevalence in hospitals was hospital-acquired while $75.7 \%$ of pressure ulcer period prevalence in nursing homes was nursing home-acquired. A longitudinal study is needed to clarify the relationship between different quality indicators of pressure ulcers and the increase/decrease of the pressure ulcer rate in response to the use of these quality indicators.
\end{abstract}

Keywords: Quality Indicators, Pressure Ulcer, Prevalence, Hospitals, Nursing Homes

\section{Introduction}

Pressure ulcers are often preventable adverse events. However, it is still a problem in many hospital settings despite the availability of best practice guidelines, pressurerelieving equipment and staff education ${ }^{(1)}$. Moreover, although pressure ulcers are becoming recognized as a largely preventable adverse event, they remain a major problem for patients in all healthcare settings and can lead to increased morbidity, particularly for the chronically ill and the elderly ${ }^{(2)}$. Furthermore, in some instances, pressure ulcers are associated with pain, fluid leakage and smell, discomfort, and difficulties with mobility; they have a profound impact on patients' lives, physically, socially, emotionally and mentally ${ }^{(3)}$.
Various pressure ulcer prevalence rates have been reported from surveys in healthcare institutions worldwide. In the United States large data sets show pressure ulcer prevalence between $14 \%$ and $17 \%{ }^{(4)}$ and the overall prevalence across five European countries (Belgium, Italy, Portugal, the United Kingdom and Sweden) was $18 \%$, ranging from $8.3 \%$ to $23 \%$ (5). Moreover, the prevalence of pressure ulcers among at-risk patients in nursing homes was $30.8 \%$ in the Netherlands and $8.3 \%$ in Germany, however, in hospitals; the prevalence in atrisk patient was $26.1 \%$ in the Netherlands and $21.2 \%$ in Germany $^{(6)}$.Additionally, a prevalence study in a Swedish university hospital revealed a pressure ulcer prevalence of $27 \%{ }^{(7)}$.

The risk factors most frequently reported as independent predictors of pressure ulcer development include three 
primary domains of mobility/activity, perfusion (including diabetes) and skin/pressure ulcer status. Skin moisture, age, hematological measures, nutrition and general health status are also important, but did not emerge as frequently as the three main domains. Overall there is no single factor which can explain pressure ulcer risk, but rather a complex interplay of factors which increase the probability of pressure ulcer development ${ }^{8}$. However the most common factors linked to pressure ulcers in hospitals and nursing homes were older age and bedridden or inactive patients ${ }^{(9,7)}$.

Pressure ulcers that developed in healthcare settings constitute one of the most important indicators of effective nursing practice ${ }^{(10)}$ and are considered to be a nursing quality indicator as reported by the California Nursing Outcomes Coalition (CalNOC), the US' largest regional nursing quality measurement network ${ }^{(11)}$. Quality of care is defined as "the degree to which health services for individuals and populations increase the likelihood of desired health outcomes and are consistent with current professional knowledge ${ }^{\text {(12) }}$. Quality of care is considered to be one of many global healthcare concerns which consequently results in quality indicators ${ }^{(13)}$. Quality indicators are used to detect optimal care either in structure, process or outcome, and can be used as a tool to guide the process of quality improvement in healthcare settings ${ }^{(14)}$. Therefore, the analysis of performance by assessing the mechanism of care delivery required for the provision of the indicated process may identify areas in which system-wide interventions could offer solutions to the problems of health care quality, such as improved methods for ordering and/or processing ${ }^{(15)}$.The rate of pressure ulcer development is a particularly important outcome measure when assessing the quality of nursing home care $^{(16)}$.

Monitoring healthcare quality makes hospital care more transparent for staff, hospitals and patients. Furthermore, it provides information that can be used to target quality improvement initiatives. However, having collections of indicator data also implies an administrative burden for staff and hospitals, and for this reason the use of this information should be optimized for hospitals ${ }^{(17)}$ as well as for nursing homes ${ }^{(9)}$. In European countries extensive studies regarding pressure ulcers have been conducted, however little is known about the Austrian prevalence and or incidence. It is therefore of the utmost importance to do research regarding different aspects of the pressure ulcer problem in Austrian settings. The present study aims to assess the existence of pressure ulcer quality indicators in Austrian hospitals and nursing homes as well as the period prevalence of hospital- and nursing home-acquired pressure ulcers to describe the magnitude of the problem in Austria

\section{Objectives}

Assess the existence of pressure ulcer quality indicators in Austrian hospitals and nursing homes.

Assess the period prevalence of both hospital- and nursing home-acquired pressure ulcers.

\section{Methodology}

\subsection{Design}

For the purposes of the present study, the German version of the Dutch National Prevalence Measurement of Care Problems (Landelijke Prevalentiemeting Zorgproblemen/LPZ) ${ }^{(18,19,20)}$ was used. The LPZ uses a multi-centre; cross-sectional design and the questionnaire were developed and have been continuously improved by the LPZ project team. It was introduced in Austria in 2009 by collecting data about quality indicators of pressure ulcer, prevention and intervention into pressure ulcers, incontinence and malnutrition. Care dependency, falls, intertrigo and restraints were also assessed. The present study focuses on the period prevalence of Austrian hospital- and nursing home-acquired pressure ulcers based on surveys conducted between 2009 and 2012 .

\subsection{Subjects}

Austrian hospitals $(n=83)$ and nursing homes $(n=40)$ with more than 50 beds were invited to participate in the study by means of leaflets and information sessions. Informed patients over the age of 18 and residents $(n=9,861 ; 3,577)$ were included in the study. Ethical approval was obtained from the university ethics committee.

\subsection{Methods}

An internal coordinator was responsible for the measurement within each participating hospital. The researchers in the study trained these coordinators and provided them with training materials, after which each coordinator, in turn, trained the teams of ward nurses on how to correctly conduct the survey and gather the data. On the day specified for the measurement, the trained nurses examined each patient in the participating hospitals. To achieve an objective assessment of every patient, two nurses (one from the patient's ward and one from a different ward) assessed the patients. Every year in April, (2009, 2010, 2011, 2012), data were collected on a previously specified day by means of a printed questionnaire which was subsequently entered into an online program.

\subsection{Tools}

The Dutch National Prevalence Measurement of Care Problems (Landelijke Prevalentiemeting Zorgproblemen/LPZ), which was used for data collection in this study, was professionally translated and sent to Austrian experts for feedback. In November 2008, a pilot study was performed in eleven Austrian hospitals to test its applicability and comprehensibility, after which the participating experts were asked for their feedback on the questionnaire. In addition, the Care Dependency Scale was incorporated into the questionnaire (21). The LPZ questionnaire covers data from hospital patients and nursing home residents. On a patient and a resident level, demographic characteristics such as sex, age, diagnosis (according to the International Classification 
of Diseases, tenth revision), care dependency, risk for pressure ulcer using Braden scale, existence of a pressure ulcer, weight, height, nutritional intake, body mass index and undesired weight loss were measured. Care dependency was assessed using the psychometrically-tested Care Dependency Scale ${ }^{(22,21)}$.Malnutrition prevalence was operationalized and validated by Meijers et al. ${ }^{(19,23)}$. Incontinence was assessed regarding type, onset, duration, the occurrence of resulting injuries as well as nursing interventions.

\subsection{Statistical Analyses}

Statistical analyses were performed with SPSS version 20 (SPSS Inc., Chicago, IL, USA). Differences between groups were tested using chi-square tests, variance analyses (ANOVA) andt-tests (for the independent sample).P-values were based on two-sided tests, and the cutoff point for statistical significance was less than 0.05 .

\section{Results}

This study was conducted on a total of 13,438 participants; $9,861(73.4 \%)$ hospital patients from with a mean age of $64 \pm$ (17.95) and 3,577 (26.4\%) nursing home residents with a mean age of $83 \pm(10.38)$. The period prevalence of pressure ulcers excluding category one in at-risk patients was $6.4 \%$ in hospitals and $6.3 \%$ in nursing homes. However, the period prevalence of hospital-acquired pressure ulcers excluding category one inat-risk patients was $2.4 \%$ and the period prevalence of nursing home-acquired pressure ulcers excluding category one was $4.6 \%$. Moreover, the period prevalence of pressure ulcers including category one in atrisk patients was nearly the same in hospitals and nursing homes. The period prevalence of hospital- and nursing homeacquired pressure ulcers includingcategory one in at-risk patients was also nearly the same in both settings (see table 1). Additionally, the prevalence of hospital- and nursing home-acquired pressure ulcers excluding category one was $4.3 \%$ in 2009 and changed slightly in 2012 to $4.6 \%$ in hospitals. However it changed from $3.4 \%$ in 2009 to $4.9 \%$ in nursing homes (see figure 1). Factors linked to pressure ulcer prevalence in nursing homes and hospitals were care dependency, Braden scale score and body mass index, while only age significantly correlated with hospital-acquired pressure ulcers (see table 2). Figure two shows that more than one quality indicator exists in both settings, namely a pressure ulcer prevention committee, pressure ulcer agreement guidelines, management protocol/guidelines for pressure ulcer prevention products, meetings held for caregivers to discuss prevention and treatment of pressure ulcers, in addition to regularly updating the prevention and treatment protocol/guidelines for pressure ulcers. The information brochure did not exist in nursing homes in 2009, however, only in hospitals (See table 3).

Table 1. Pressure ulcer period prevalence and acquired period prevalence in hospitals and nursing homes

\begin{tabular}{lllll}
\hline & ${ }^{1}$ PU Prevalence & & \multicolumn{2}{l}{ Acquired ${ }^{\mathbf{P} U}$ Prevalence } \\
\cline { 2 - 5 } & HospitalsN (\%) & Nursing homesN (\%) & HospitalsN (\%) & Nursing homesN (\%) \\
\hline${ }^{1}$ PU at-risk group including grade 1 & $296(10.2 \%)$ & $200(7.4 \%)$ & $22(4.5 \%)$ & $27(5.6 \%)$ \\
${ }^{1}$ PU at-risk group excluding grade 1 & $186(6.4 \%)$ & $169(6.3 \%)$ & $69(2.4 \%)$ & $102(4.6 \%)$ \\
\hline
\end{tabular}

${ }^{1}$ Pressure Ulcer

Table 2. Factors associated with hospital- and nursing home-acquired pressure ulcers

\begin{tabular}{|c|c|c|c|c|c|c|c|c|}
\hline \multirow{3}{*}{ Factors } & \multirow{3}{*}{$\begin{array}{l}\text { Hospitals } \\
\text { Mean } \pm \mathbf{S D}^{1}\end{array}$} & & & & \multicolumn{4}{|c|}{ Nursing homes } \\
\hline & & \multirow{2}{*}{ P-Value } & \multicolumn{2}{|c|}{ Confidence interval } & \multirow{2}{*}{ Mean \pm SD } & \multirow{2}{*}{ P-Value } & \multicolumn{2}{|c|}{ Confidence interval (CI) } \\
\hline & & & Lower & Upper & & & Lower & Upper \\
\hline Age & $76 \pm(11.83)$ & 0.01 & -6.97 & 1.17 & $82 \pm(10.91)$ & 0,479 & -1.29 & 2.73 \\
\hline Body mass index & $26.7 \pm(7.26)$ & 0.295 & -2.75 & 0.84 & $24.6 \pm(5.50)$ & 0.000 & 0.98 & 3.02 \\
\hline Care dependency scale & $41.4 \pm(18.77)$ & 0.000 & 7.96 & 17.06 & $32.8 \pm(14.90)$ & 0.000 & 4.74 & 9.19 \\
\hline BradenScale & $13.9 \pm(3.44)$ & 0.000 & 2.19 & 3.86 & $15 \pm(3.07)$ & 0.000 & 2.35 & 3.61 \\
\hline
\end{tabular}

1, Standard Deviation.

Table 3. Existence of pressure ulcer quality indicators in hospitals and nursing homes

\begin{tabular}{|c|c|c|c|c|c|c|c|c|}
\hline \multirow[b]{2}{*}{ Quality indicator } & \multicolumn{2}{|l|}{2009} & \multicolumn{2}{|l|}{2010} & \multicolumn{2}{|l|}{2011} & \multicolumn{2}{|l|}{2012} \\
\hline & $\begin{array}{l}\text { Hospitals } \\
\text { N (11) }\end{array}$ & $\begin{array}{l}\text { Nursing } \\
\text { homes N (6) }\end{array}$ & $\begin{array}{l}\text { Hospitals } \\
\text { N (18) }\end{array}$ & $\begin{array}{l}\text { Nursing } \\
\text { homesN (18) }\end{array}$ & $\begin{array}{l}\text { Hospitals } \\
\text { N (21) }\end{array}$ & $\begin{array}{l}\text { Nursing } \\
\text { homesN (9) }\end{array}$ & $\begin{array}{l}\text { Hospitals } \\
\text { N (33) }\end{array}$ & $\begin{array}{l}\text { Nursing } \\
\text { homesN (7) }\end{array}$ \\
\hline $\begin{array}{l}\text { Pressure ulcer prevention } \\
\text { committee }\end{array}$ & $\begin{array}{l}8 \\
(72.7 \%)\end{array}$ & $\begin{array}{l}5 \\
(83.3 \%)\end{array}$ & $\begin{array}{l}14 \\
(77.8 \%)\end{array}$ & $\begin{array}{l}9 \\
(50 \%)\end{array}$ & $\begin{array}{l}9 \\
(42.9 \%)\end{array}$ & $\begin{array}{l}7 \\
(77.8 \%)\end{array}$ & $10(30.3 \%)$ & $\begin{array}{l}3 \\
(42.9 \%)\end{array}$ \\
\hline $\begin{array}{l}\text { Pressure ulcer agreement } \\
\text { guidelines }\end{array}$ & $\begin{array}{l}10 \\
(90.9 \%)\end{array}$ & $\begin{array}{l}5 \\
(83 \%)\end{array}$ & $\begin{array}{l}17 \\
(94.4 \%)\end{array}$ & $\begin{array}{l}7 \\
(38.9 \%)\end{array}$ & $11(52.4 \%)$ & 7 & $10(30.3 \%)$ & 7 \\
\hline $\begin{array}{l}\text { Management protocol for } \\
\text { pressure ulcer prevention } \\
\text { products }\end{array}$ & $\begin{array}{l}7 \\
(63.6 \%)\end{array}$ & $\begin{array}{l}4 \\
(66.7 \%)\end{array}$ & $\begin{array}{l}8 \\
(44.4 \%)\end{array}$ & $\begin{array}{l}5 \\
(27.8 \%)\end{array}$ & $\begin{array}{l}7 \\
(33.3 \%)\end{array}$ & $\begin{array}{l}6 \\
(66.7 \%)\end{array}$ & $19(57.6 \%)$ & $\begin{array}{l}7 \\
(100 \%)\end{array}$ \\
\hline Information brochure & $\begin{array}{l}1 \\
(9 \%)\end{array}$ & - & $\begin{array}{l}1 \\
(5.6 \%)\end{array}$ & $\begin{array}{l}2 \\
(11 \%)\end{array}$ & $\begin{array}{l}5 \\
(23.8 \%)\end{array}$ & $\begin{array}{l}1 \\
(11 \%)\end{array}$ & $\begin{array}{l}5 \\
(15.2 \%) \\
\end{array}$ & $\begin{array}{l}2 \\
(28.6 \%)\end{array}$ \\
\hline
\end{tabular}




\begin{tabular}{|c|c|c|c|c|c|c|c|c|}
\hline \multirow[b]{2}{*}{ Quality indicator } & \multicolumn{2}{|l|}{2009} & \multicolumn{2}{|l|}{2010} & \multicolumn{2}{|l|}{2011} & \multicolumn{2}{|l|}{2012} \\
\hline & $\begin{array}{l}\text { Hospitals } \\
\text { N (11) }\end{array}$ & $\begin{array}{l}\text { Nursing } \\
\text { homes N (6) }\end{array}$ & $\begin{array}{l}\text { Hospitals } \\
\text { N (18) }\end{array}$ & $\begin{array}{l}\text { Nursing } \\
\text { homesN (18) }\end{array}$ & $\begin{array}{l}\text { Hospitals } \\
\text { N (21) }\end{array}$ & $\begin{array}{l}\text { Nursing } \\
\text { homesN (9) }\end{array}$ & $\begin{array}{l}\text { Hospitals } \\
\text { N (33) }\end{array}$ & $\begin{array}{l}\text { Nursing } \\
\text { homesN (7) }\end{array}$ \\
\hline $\begin{array}{l}\text { Meetings for caregivers to } \\
\text { discuss prevention and } \\
\text { treatment of pressure ulcers }\end{array}$ & $\begin{array}{l}10 \\
(90.9 \%)\end{array}$ & $\begin{array}{l}6 \\
(100 \%)\end{array}$ & $\begin{array}{l}16 \\
(88.9 \%)\end{array}$ & $\begin{array}{l}15 \\
(83.3 \%)\end{array}$ & $13(61.9 \%)$ & $\begin{array}{l}8 \\
(88 \%)\end{array}$ & $14(42.2 \%)$ & $\begin{array}{l}6 \\
(85.7 \%)\end{array}$ \\
\hline $\begin{array}{l}\text { Regular updating of the } \\
\text { prevention and treatment } \\
\text { protocol for pressure ulcers. }\end{array}$ & $\begin{array}{l}9 \\
(81.8 \%)\end{array}$ & $\begin{array}{l}5 \\
(83.3 \%)\end{array}$ & $\begin{array}{l}16 \\
(88.9 \%)\end{array}$ & $\begin{array}{l}5 \\
(27.8 \%)\end{array}$ & $\begin{array}{l}9 \\
(42.9 \%)\end{array}$ & $\begin{array}{l}6 \\
(66.7 \%)\end{array}$ & $\begin{array}{l}9 \\
(27.3 \%)\end{array}$ & $\begin{array}{l}7 \\
(100 \%)\end{array}$ \\
\hline
\end{tabular}

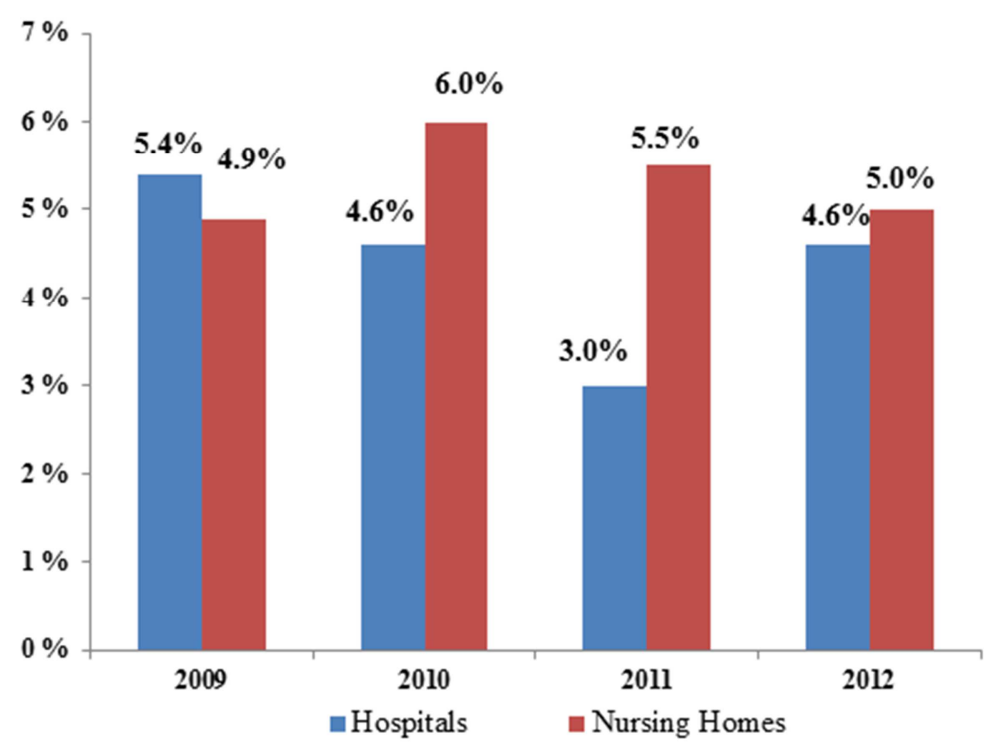

Figure 1. Prevalence of hospital- and nursing home-acquired pressure ulcers

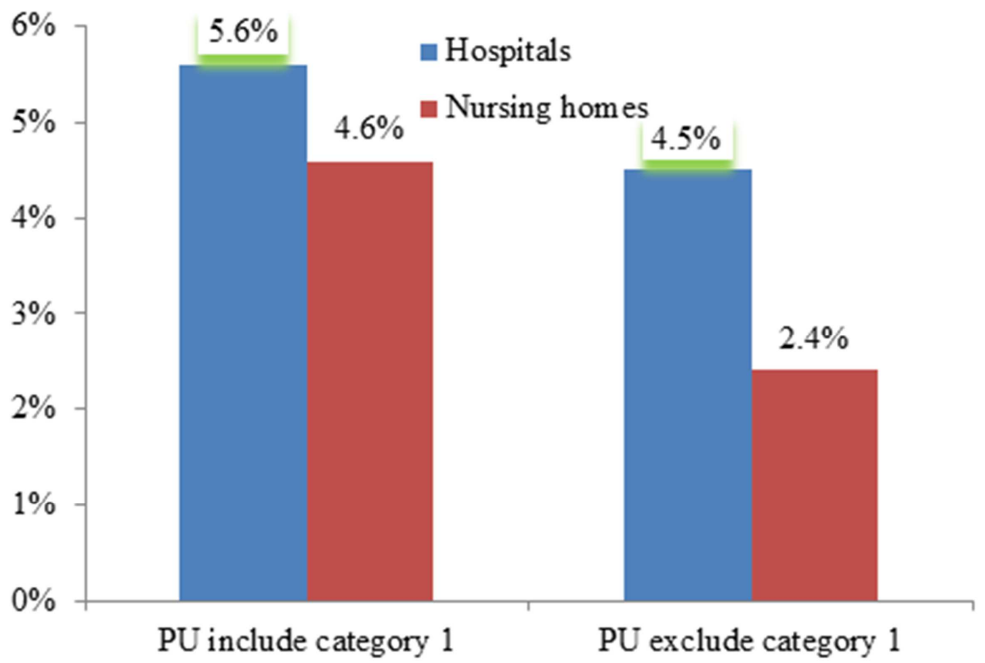

Figure 2. Institution-acquired prevalence in hospital patients and nursing homes residents at PU risk (Bradenscale) in \%

\section{Discussion}

The study results revealed a similar period prevalence of pressure ulcers in at-risk patients including category one in hospitals and nursing homes. However, the prevalence of hospital-acquired pressure ulcers excluding category one changed slightly from $4.3 \%$ in 2009 to $4.6 \%$ in 2012 . Whereas, nursing home-acquired pressure ulcers excluding category one increased from $3.4 \%$ in 2009 to $4.9 \%$ in 2012 . Although more than one quality indicator exists in hospitals and nursing homes, (e.g. a pressure ulcer prevention committee, pressure ulcer agreement guidelines, and management protocol/guidelines for pressure ulcer prevention products) nursing home-acquired pressure ulcer prevalence negatively changed over a four year-period (2009 to 2012) in nursing homes and increased slightly in hospitals, however, a reduction in pressure ulcer prevalence in both healthcare settings might be expected. In a longitudinal study from 2008 to 2011 in neurosurgery and neurology units, more than one preventive measure for pressure ulcers was applied, namely 
turning and positioning patients every $2 \mathrm{~h}$, utilizing specialty beds when necessary, and forming a "skin and wound assessment team" consisting of two "expert" nurses, and two nursing assistants who would see all neuroscience patients at least once a week, and attend monthly conferences the hospital team. These preventative measures achieved a $48 \%$ reduction in hospital-acquired pressure ulcers in the intensive care unit, and a 59\% reduction on the floor; these reductions were not only maintained but improved upon by 2010 (intensive care unit $57 \%$ reduction, $65 \%$ floor reduction), and 2011 (intensive care unit reduction $61 \%, 82 \%$ floor reduction) ${ }^{(24)}$.

Furthermore, Barker et al. conducted a 9-year study involving a review of 4,268 hospital charts. The authors assessed the prevalence of hospital-acquired pressure ulcers by utilizing three best practice guidelines (validated pressure ulcer risk assessment and intervention checklist, demonstrating accuracy of risk assessment scoring through usual nursing staff versus experienced injury/ulcer prevention nurses and the use of pressure ulcer prevention strategies) which revealed hospital-acquired pressure ulcer reduction from $12.6 \%$ in 2003, two years before program implementation, to $2.6 \% 6$ years after program implantation. Interestingly, compliance with the guidelines from the nursing staff was over $84 \%{ }^{(1)}$. In contrast to Baker et al., a study in a Swedish hospital revealed that pressure ulcer prevalence including category one was $23.9 \%$ in 2002 and $22.9 \%$ in 2006 , and the prevalence did not decrease despite a comprehensive quality improvement program. In the same study, however, the prevalence of pressure ulcers excluding category one increased from $8 \%$ in 2002 to $12 \%$ in $2006^{(25)}$ and these result are consistent with the current study results. Additionally, in a literature review study about prevalence and incidence of pressure ulcer in intensive care setting revealed that more research on pressure ulcers in intensive care settings is needed regarding different aspects such as the gap between theory and practice, the effectiveness of nursing care on pressure ulcer development and the requirements for and the qualification of nurses specialized in pressure ulcer prevention and treatment in intensive care settings ${ }^{(26)}$.

At the same time, the increase of nursing home-acquired pressure ulcers in Austria from 2009 to 2012couldrefer to the length of the resident's stay in the nursing home and this stay in combination with more than one risk factor, such as a lower Braden score, decreased body mass index and decreased level of care dependency. This could lead to more pressure ulcer development and a negative prognosis from pressure ulcer grade one to as high as grade two or more. Additionally old age could be associated with decreased immunity and deceased level of wound healing and this might be another reason for increased pressure ulcer prevalence excluding category one. This is consistent with the 2012report fromAgency for Healthcare Research and Quality (AHRQ), which stated that one of the top predictors of developing a new ulcer is a patient's inability to move independently. In addition to other factors such as needing assistance with grooming and dressing, dependence in toileting, being bedridden, and already having a pressure ulcer at the start of home healthcare ${ }^{(27)}$. Moreover, a more recent review that included 54 studies and covered 34,449 patients from acute and community patient populations stated that no single factor can explain pressure ulcer risk, rather a complex interplay of factors increase the probability of pressure ulcer development ${ }^{(8)}$.

Regarding factors linked to acquired pressure ulcer prevalence in nursing homes, a recent study was consistent with the results of the current study and revealed that being bedridden, and having a low body mass index were linked to pressure ulcers in nursing homes in addition to old age ${ }^{(9)}$. However the current study found that old age was not significantly associated with acquired pressure ulcer prevalence in nursing homes but it was in hospitals. This could be referring to the fact that the mean age of nursing home residents was higher than 80 years, thus limiting the difference in residents' ages, while the mean age in hospital patients was 64 years, allowing for more age variation in the hospitals. Otherwise the current study revealed that a low Braden score, low body mass index and low level of care dependency were significantly linked to hospital- and nursing home-acquired pressure ulcers. The study by Wann-Hansson \& Willman concurs with these results and found that a Braden score of $<17$, old age and inactivity were significantly associated with hospital-acquired pressure ulcers ${ }^{(7)}$.

\section{Conclusion}

The study analysis concluded that less than half of pressure ulcer prevalence excluding category one in hospitals constitutes an acquired hospital pressure ulcer and more than seventy five percent of pressure ulcer prevalence excluding category one in nursing homes constitutes an acquired nursing home pressure ulcer. The prevalence of pressure ulcers in nursing homes increased from 2009 to 2012 and slightly increased over the same years in hospitals. Positive changes in hospital-acquired pressure ulcers might be a reflection of the use of quality indicators such as prevention and/or treatment protocol/guidelines. However, the negative changes in nursing home-acquired pressure ulcers could be referring to the inappropriate application of the quality indicators and/or the fact that nursing home residents are more susceptible to pressure ulcer development. More than one quality indicator exists in both settings. A longitudinal study is needed to clarify the relationship between different quality indicators for pressure ulcers and the changes that occurred in pressure ulcer rates as a response to the use of these quality indicators.

\section{Study Limitations}

The prevalence design is only a snapshot in spite its continuous use over four years, and therefore could be influenced by discharge practices. For example, a hospital that is able to quickly discharge patients with a pressure ulcer to a nursing home, even before it is apparent, may have a lower prevalence of pressure ulcers than a hospital that can 
only discharge patients after the pressure ulcer has healed. If the prevention and treatment protocols are of low quality or there is a low compliance rate, then it is likely that both the prevention and treatment of pressure ulcers will be suboptimal. This may lead to patients experiencing their pressure ulcers for a longer period of time ${ }^{(28)}$. Additionally, the questionnaire part regarding quality indicators was made up of dichotomized questions and thus recorded only the presence of quality indicators but not whether or not they were followed by staff. Therefore the questionnaire cannot give an accurate explanation of negative or positive changes in pressure ulcer prevalence in relation to the existence of pressure ulcer quality indicators. However, the study provided important insights on the topic.

\subsection{Strengths}

1. This article covered large sample size (number of patients) and multiple study centers (hospitals and nursing homes).

2. The study design is period prevalence and it is accurate more than point prevalence.

3. Tools used in methodology and analysis are multiple and accurate.

4. Most of references were up to date and statistical analysis conducted using newversion of statistical program and based on newer hypothesis

Tests are good enough to analyze and compare two groups.

\subsection{Weakness}

1. The prevalence design is only a snapshot in spite its continuous use over four years, and therefore could be influenced by discharge practices. For example, a hospital that is able to quickly discharge patients with a pressure ulcer to a nursing home, even before it is apparent, may have a lower prevalence of pressure ulcers than a hospital that can only discharge patientsafter the pressure ulcer has healed. If the preventionand treatment protocols are of low quality or there is a low compliance rate, then it is likely that both the prevention and treatment of pressure ulcers will be suboptimal. This may lead to patients experiencing their pressure ulcers for a longer period of time.

The study did not cover different types of health care facilities as intermediate care or rehabilitation centers.

\section{Suggestions on How to Improve the Paper}

1. Longitudinal (incidence) or quasi experimental study will give more accurate magnitude of pressure ulcer development in addition to relevant factors associated with it.

2. Detail assessment of nursing practice regarding preventive care of pressure ulcer from patient admission until discharge or transfer.

3. The study will be more prevalent if extended to cover more hospital categories and specific wards as intermediate care and different types of surgical words as orthopedic surgeries and open heart surgery.

\section{Acknowledgements}

We appreciate and gratefully thank all hospital- and nursing home administrators as well as hospital patients and nursing home residents who agreed to participate in this study.

\section{References}

[1] Barker AL, Kamar J, Tyndall TJ, White L, Hutchinson A, Klopfer $\mathrm{N}$, Weller $\mathrm{C}$, Implementation of pressure ulcer prevention best practice recommendations in acute care: an observational study. Int Wound J. 2013; 10:313-320.

[2] Lyder C, Pressure ulcer prevention and management. JAMA 2003;289:223-6.

[3] Spilsbury K, Nelson A, Cullum N, Iglesia C, Nixon J \& Mason S, Pressure ulcers and their treatment and effects on quality of life: hospital inpatient perspectives. J Adv Nurs 2007; 57: 494-504

[4] Whittington $\mathrm{K} \mathrm{T}$, Briones $\mathrm{R}$, National Prevalence and Incidence Study: 6-year sequential acute care data. Adv Skin Wound Care 2004;17: 490-494.

[5] Vanderwee K, Clark M, Dealey C, Gunningberg L, Defloor T, Pressure ulcer prevalence in Europe: a pilot study. J Eval Clin Pract 2007; 13: 227-235.

[6] Tannen A, Dietz E, Dassen T, Halfens R,Explaining the national differences Inpressure ulcer prevalence between the Netherlands and Germany -adjusted For personal risk factors and institutional quality indicators. JEval Clin Pract 2009; 15: $85-90$

[7] Wann-Hansson C, Hagell P, Willman A, Risk factors and prevention among patients with hospital-acquired and preexisting pressure ulcers in an acute care hospital. J Clin Nurs $2007 ; 17: 1718-1727$

[8] Coleman S, Gorecki C, Nelson EA, et al, Patient risk factors for pressure ulcer development: Systematic review.Int J Nurs Stud 2013; 50: 974-1003

[9] Temkin-Greener H, Cai S, Zheng NT, Zhao H, Mukamel DB, NursingHomeWork Environment and the Risk of Pressure Ulcers and Incontinence. Health Serv Res 2012; 47: 1180 - 1200

[10] Benbow M, Bateman S, Working towards clinical excellence Pressure ulcer prevention and management in primary and secondary care. J Wound Care 2012; 21: S25 - S40.

[11] Aydin C E, Bolton LB, Donaldson N, Brown D, Mukerji A. Beyond nursingQuality measurement: the nation's first regional nursing virtual dashboard. InAdvances in Patient Safety: New Directions and Alternative Approaches. 2008; Volumes 1-4,AHRQ Publication Nos. 08-0034 (1-4). July 2008. Agency for Healthcare Research and Quality, Rockville, MD.http://www.ahrq.gov/qual/advances2/

[12] Glickman SW, Baggett KA, Krubert CG, Peterson ED, Schulman KA, Promoting quality: the health-care organization from a management perspective.IntJ Qual Health Care 2007; 19: $341-348$. 
[13] Lena- Gunningberg, L., Donaldson N, Aydin C, Ewa Idvall E, Exploring variation in pressure ulcer prevalence in Sweden and the USA: benchmarking in action. JEval Clin Pract2012; j18: 904-910 ep

[14] Donabedian A. Explorations in Quality Assessment and Monitoring: TheDefinition of Quality and Approaches to Its Assessment. Michigan: Health Administration Press 1980.

[15] McGlynn EA, Asch SM, Adams J, Keesey J, Hicks J, DeCristofaro A, Kerr EA, The Quality of Health Care Delivered to Adults in the United States. N Eng J Med 2003; 348: $2635-2645$.

[16] Berlowitz, D. R., G. H. Brandeis, J. N. Morris, et al, Deriving a Risk-Adjustment Model for Pressure Ulcer Development Using the Minimum Data Set. JAGS 2001; 49: 866-71.

[17] De vos M, Graafmans W, Kooistra M, Meijboom B., et al,Using quality indicators to improve hospital care: a review of the Literature. Int J Qualy Health Care2009;21: 119-129

[18] Halfens RJG, Janssen MAP, Meijers JMM \& Wansink SW. Landelijke Prevalentiemeting Decubitus en andere zorgproblemen: herziene resultatenzevende jaarlijkse meting 2004. Universiteit Maastricht, Maastricht, ISBN 90-806663-51.

[19] Meijers JMM, Schols JMGA, Jackson PA. et al. Differences in nutritional care in pressure ulcer patients whether or not using nutritional guidelines. Nutrition 2008; 24: 127-132

[20] Lohrmann C. P rävalenzerhebung 07. April 2009. Institut für Pflegewissenschaft, Medizinische Universität Graz.
[21] Lohrmann C. Die Pflegeabhängigkeitsskala: ein Einscha"tzungsinstrument für Heime und Kliniken - Eine methodologische Studie, PhD Thesis, 2003, CentreofHumanities and Health Science, ChariteUniversita"tsmedizin, Berlin, Germany.

[22] Dijkstra A. Care Dependency - An Assessment Instrument for Use in Long-term Care Facilities, PhD Thesis 1998; Rijksuniversiteit Groningen,Groningen, The Netherlands.

[23] Meijers JMM, Schols JMGA, Van Bokhorstde van der Schueren MAE, Dassen T,Janssen MAP, Halfens RJG, Malnutrition prevalence in the Netherlands: Results of the annual Dutch National Prevalence Measurement of Care Problems.BrJ Nutr 2009; 101: 417-423.

[24] McGuinness J, Persaud-Roberts S, Marra S, Ramos J, Toscano D, Policastro L, and Epstein NE, How to reduce hospital-acquired pressure ulcers on aneuroscience unit with a skin and wound assessment team. Surg Neurol Int 2012; 3:773 -777 .

[25] Gunningberg L and Stotts NA, Tracking quality over time: what do pressure ulcer data show? Int Qual Health Care. 2008;20 (4): 246-253.

[26] Pressure ulcer prevalence and incidence in intensive care pts.: a literature review. Nursing in critical care. 2008;13(2): 71-79

[27] Agency for Healthcare Research and Quality AHRQ. Bowel incontinence and other clinical pressure ulcers. 2012; 18.(384).

[28] European pressure ulcer advisory panel (EPUAP). Prevalence and incidence Monitoring of pressure ulcers. 2005; 6: 69-84. 